\title{
PRODUÇÃO DE MASSA SECA E ÁREA FOLIAR DO AÇAIZEIRO SOB DÉFICIT HÍDRICO
}

\author{
Clivia da Conceição Mar¹ Heráclito Eugenio Oliveira da Conceição²; Ana Brígida \\ Rabelo dos Santos ${ }^{3}$; Ismael de Jesus Matos Viégas ${ }^{4}$; Francisco Sergio Neres da Silva ${ }^{5}$. \\ ${ }^{1}$ Universidade Federal Rural da Amazônia. Capitão Poço, Pará, Brasil. cliviahmar@ hotmail.com \\ ${ }^{2}$ Universidade Federal Rural da Amazônia. Belém, Pará, Brasil. agroheraclito@ yahoo.com.br \\ ${ }^{3}$ Secretaria Executiva de Educação do Estado do Pará. Belém, Pará, Brasil. brigisan@ yahoo.com.br \\ ${ }^{4}$ Universidade Federal Rural da Amazônia. Capanema, Pará, Brasil. ismael.viegas@ hotmail.com \\ ${ }^{5}$ Universidade Federal Rural da Amazônia. Capitão Poço, Pará, Brasil.
}

RESUMO: O presente trabalho teve como objetivo avaliar a produção de massa seca e a área foliar em plantas jovens de açaizeiro (Euterpe oleracea Mart.) quanto à disponibilidade da água no solo, especialmente na fase de produção de mudas. O experimento foi conduzido em condições de casa de vegetação localizada na Embrapa Amazônia Oriental, em Belém, Pará. Utilizaram-se mudas de açaizeiros produzidas a partir de sementes, em vasos de plástico com capacidade para $10 \mathrm{~kg}$ de substrato. A imposição dos tratamentos para a espécie ocorreu quando as plantas apresentavam-se com seis meses de idade. Foram usados os seguintes tratamentos: a) controle e b) estressado. Foram avaliados os seguintes parâmetros: a) área foliar e b) pesos de massas secas de folíolo, pecíolo, ráquis, bainha, ápice caulinar e sistema radicular. O delineamento experimental usado foi inteiramente casualizado com três repetições. Os resultados obtidos nesse trabalho, com plantas jovens de açaizeiros submetidos a ciclos de déficits hídricos de diferentes intensidades evidenciaram que, as produções de massas secas de raízes, folhas, folíolos, ráquis, pecíolos, bainhas foram reduzidas significativamente.

PALAVRAS-CHAVE: Euterpe oleracea, crescimento e estresse hídrico.

\section{DRY MASSES AND LEAF ÁREA PRODUCTION OF YOUNG AÇAÍ PALM SUBMITTED TO WATER DEFICITS}

\begin{abstract}
The present work had as objective to evaluate the production of dry mass and the leaf area in the young plants to açai palm (Euterpe oleracea Mart.) with relationship to the readiness of the water in the soil, especially in the production cuttings phase. The experiment was driven in conditions of greenhouse in Embrapa Amazônia Oriental, in Belém, Pará. Açai palm cuttings cultivated in vases of plastic with capacity for $10 \mathrm{~kg}$ of substratum. After the transplanted, whole the plants were irrigated daily, being looked for to maintain the humidity of the close soil a field capacity. The imposition of the treatments happened when the plants came with six months of age. The following treatments were used: a) control e b) stressed. They were appraised the following parameters: a) leaf area and b) weights of dry masses of leaves, leaflets, petioles, rachs, sheaths, shoot apex and system root. The experiment was carried out in random way, with three replications. The results obtained in that work, with young plants of açai palm submitted the cycles of water deficits of different intensities evidenced that, the productions of dry masses of system root, leaves, leaflets, rach, petioles and sheats were significantly reduced.
\end{abstract}

KEYWORDS: Euterpe oleracea, growth and water stress. 


\section{INTRODUÇÃO}

$\mathrm{O}$ açaizeiro, Euterpe oleracea Mart., é uma palmácea nativa da Amazônia, fruteira de grande potencial econômico, que ocorre em grandes extensões no estuário amazônico e em áreas de terra firme, principalmente quando localizadas próximas às várzeas e igapós (LORENZI et al., 1996; JARDIM, 2002; VIEGAS et al., 2004). Constitui o alimento principal para as populações ribeirinhas, com inúmeras utilidades na indústria alimentícia (sorvetes, licores, doces, torrefação de café), farmacêutica (corantes, cosméticos) carvão vegetal, adubo orgânico entre outros (SUFRAMA, 2003; TINOCO, 2005).

A valorização do fruto trouxe benefício econômico e ecológico para a população regional promovendo a conservação das palmeiras de açaí. Com a conquista de novos mercados e tornou-se uma importante fonte de renda e de emprego. A polpa congelada é exportada também na forma de mix, para outros estados brasileiros e outros países, ultrapassando mil toneladas por ano (IBGE, 2012).

Entretanto, apesar da grande divulgação em nível nacional e internacional e, do potencial econômico apresentado atualmente por essa espécie, ainda existem poucos estudos com enfoque nos processos de crescimento e desenvolvimento dessa planta frente a déficits hídricos.

A ocorrência sazonal de baixa disponibilidade de água no solo é considerada uma das mais importantes condições de estresse ambiental, capaz de influenciar de maneira significativa no crescimento, desenvolvimento e na sobrevivência das plantas (SMIT, 1992).

A falta de água no solo limita intensamente o crescimento das espécies vegetais em várias regiões do mundo, tornando-se limitante principalmente nos períodos de baixa pluviosidade, ocasionando efeitos deletérios que poderão afetar a produtividade (LECHINOSKI et al., 2007).

A insuficiência de água representa um estresse abiótico, sendo considerada uma das maiores limitações agrícolas, devido a influências negativas em relação ao crescimento e desenvolvimento (ENDRES et al., 2010).

De acordo com Jaleel et al. (2009) o estresse hídrico inibe o crescimento celular reduzindo o crescimento das plantas, afetando vários processos fisiológicos, como fotossíntese e respiração, bem como a produção.

Apesar de algumas espécies de palmeiras desenvolverem mecanismos de tolerância ao estresse hídrico no solo (CALBO; MORAES, 1997; CALBO; MORAES, 2000; GOMES et al., 2008; 
OLIVEIRA et al., 2002; REPELLIN et al., 1994), os estudos de fisiologia de palmeiras são escassos, principalmente no que diz respeito aos mecanismos de tolerância a esta condição.

$\mathrm{O}$ presente trabalho teve como objetivo avaliar o padrão de crescimento e o desenvolvimento de plantas jovens de açaizeiros sob déficit hídrico, por meio de técnicas de análise de crescimento de plantas, especialmente na fase de produção de mudas.

\section{MATERIAL E MÉTODOS}

$\mathrm{O}$ experimento foi conduzido $\mathrm{em}$ condições de casa de vegetação, localizada na Embrapa Amazônia Oriental, em Belém, Pará, cujas coordenadas geográficas são de 01²4'59" e 01²7'40" de latitude sul, e 48 $20^{\prime} 55^{\prime \prime}$ e $48^{\circ} 26{ }^{\prime} 59^{\prime \prime}$ de longitude oeste de Greenwich, durante o período de janeiro a agosto de 2012 .

Utilizou-se mudas de açaizeiro produzidas a partir de sementes oriundas de áreas do banco ativo de germoplasma (BAG) da Embrapa Amazônia Oriental/Belém-Pa. Quando as plantas apresentavam-se com três meses de idade foram transplantadas para vasos de plástico com capacidade para $6 \mathrm{~kg}$ substrato constituído de terra preta e serragem, na proporção volumétrica de 2:1. Foram adicionados ao substrato $2 \mathrm{~g}$ de termofosfato de yorim. Posteriormente foram realizadas duas adubações na base de $1 \mathrm{~g}$ de uréia $+1 \mathrm{~g}$ de cloreto de potássio por $1 \mathrm{~kg}$ de substrato respectivamente aos 4 e 5 meses de idade das plantas.

Após o transplantio durante o período que antecedeu a imposição dos regimes hídricos, todas as plantas foram irrigadas diariamente durante um período necessário para o seu completo estabelecimento. A imposição dos regimes hídricos para a espécie ocorreu quando as plantas apresentavam-se com seis meses de idade.

Foram usados os seguintes tratamentos: a) Controle (C) - umidade do solo mantido entre 80 a $100 \%$ da capacidade de campo e b) Estressado (E) umidade do solo reduzida por meio da suspensão da irrigação durante 25 dias no primeiro ciclo e, depois, reirrigação até atingir o nível de água do tratamento controle (C), durante cinco dias e, novamente, suspensão da irrigação durante 14 dias no segundo ciclo. A imposição dos tratamentos hídricos teve a duração de 44 dias.

Os efeitos dos tratamentos foram avaliados pelos seguintes parâmetros: a) área foliar (Af) e b) massas secas da folha (MSF), ápice caulinar (MSAC), sistema radicular (MSR), parte aérea (MSPA) e total (MST). As determinações dos parâmetros foram realizadas com $0,15,25$ e 44 dias, após a imposição dos tratamentos. Nessas épocas de avaliações, 
os valores das umidades do solo do tratamento estressado foram equivalentes a $100,0 \% ; 35,3 \% ; 24,7 \%$ e $47,4 \%$, em relação aos níveis de umidade do solo do tratamento controle.

O experimento foi conduzido segundo delineamento inteiramente casualizado, com três repetições e uma planta por parcela útil.

Os dados obtidos foram submetidos à análise de variância e, quando detectada diferença significativa, as médias foram comparadas pelo teste de Tukey a 0,05 de probabilidade, utilizando-se o programa de software Statistica, versão 6.0. (STATSOFT, 2006).

Em geral, todos os parâmetros de produção de biomassa seca e de área foliar do tratamento sob déficit hídrico foram reduzidos diferente e significativamente, em relação ao tratamento controle (Tabela 1)

\section{RESULTADOS E DISCUSSÃO}

As produções de MSF em plantas jovens de açaizeiros no tratamento controle variaram entre 6,81 e 15,2 g/planta e, no tratamento estressado de 6,61 e 12,54 $\mathrm{g} /$ planta, ao final de 44 dias de exposição dos tratamentos (Tabela 1). Observam-se aumentos significativos ( $p>0,05)$ da MSF, tanto dentro como entre tratamentos. No tratamento controle o acúmulo máximo de MSF ocorreu a partir de 25 dias e, para o tratamento estressado, esse comportamento só é evidenciado aos 44 dias. Já, entre os tratamentos, verificaram-se reduções na produção da MSF do tratamento estressado, aos 25 e 44 dias de imposição dos ciclos de déficits hídricos.

As produções de MSAC de açaizeiros jovens do tratamento controle oscilaram entre 0,26 e $0,68 \mathrm{~g} /$ planta e, no tratamento estressado entre 0,25 e $0,73 \mathrm{~g} /$ planta, ao final do período experimental (Tabela 1). Os acúmulos máximos de MSAC dentro de cada tratamento apresentaram a mesma tendência dos observados para a MSF (Tabela 1). Houve redução na produção de MSAC do tratamento estressado aos 15 e 25 dias, detectada durante o primeiro ciclo de déficit hídrico e, foram equivalentes a 42,0 e $27,2 \%$, em relação às plantas jovens de açaizeiros do tratamento controle, porém, após este período, verifica-se uma recuperação deste parâmetro.

As produções de MSR em plantas jovens de açaizeiros no tratamento controle ficaram entre 3,08 a 7,58 g/planta e, entre 3,26 e 6,06 g/planta, no tratamento estressado. Pode ser observado, na Tabela 1, a existência de efeitos significativos diferenciados $(p>0,05)$, para a produção da MSR das plantas jovens de açaizeiros do tratamento estressado da ordem de 20,8 e $20,0 \%$, respectivamente para as épocas de avaliações feitas aos 15 e 44 dias, após a imposição da restrição hídrica, em 
comparação com os valores obtidos para esta variável no tratamento controle.

É relatado por diversos autores que plantas submetidas à deficiência hídrica acumulam fotoassimilados na raiz em função da paralisação do crescimento da parte aérea. Representando assim, uma tentativa de contornar a seca por meio do acréscimo da área de absorção no solo (SILVA; NOGUEIRA, 2008;
MACHADO, 2004; FIGUEIRÔA et al., 2004).

As produtividades primárias das plantas jovens de açaizeiros do tratamento estressado, avaliadas em termos de MSPA e MST sofreram reduções da ordem de 17,8 e 19,1\%, após 25 e 44 dias e de 13,8, 15,6 e 17,6\%, determinadas após 15, 25 e 44 dias, respectivamente, em função do tratamento controle (Tabela 1).

Tabela 1. Massas secas de folíolos, pecíolos, ráquis, bainha, ápice caulinar, sistema radicular e total e, da área foliar de plantas jovens de açaizeiro, em função dos tratamentos.

\begin{tabular}{|c|c|c|c|c|}
\hline \multirow[t]{2}{*}{ Nível de água } & \multicolumn{4}{|c|}{ Épocas de avaliação (dias) } \\
\hline & 0 & 15 & 25 & 44 \\
\hline \multicolumn{5}{|c|}{ Massa seca da folha (g/planta) } \\
\hline Controle & $6,81 \mathrm{cA}$ & $10,77 \mathrm{bA}$ & $12,10 \mathrm{Aa}$ & $15,20 \mathrm{aA}$ \\
\hline Estressado & $6,61 \mathrm{cA}$ & $9,80 \mathrm{bA}$ & $9,67 \mathrm{bB}$ & $12,54 \mathrm{aB}$ \\
\hline Média & $6,71 \mathrm{~d}$ & $10,29 \mathrm{c}$ & $10,89 \mathrm{~b}$ & $13,87 \mathrm{a}$ \\
\hline \multicolumn{5}{|c|}{ Massa seca do ápice caulinar (g/planta) } \\
\hline Controle & $0,26 \mathrm{cA}$ & $0,50 \mathrm{bA}$ & $0,55 \mathrm{abA}$ & $0,68 \mathrm{aA}$ \\
\hline Estressado & $0,25 \mathrm{bA}$ & $0,29 \mathrm{bB}$ & $0,40 \mathrm{bB}$ & $0,73 \mathrm{aA}$ \\
\hline Média & $0,26 \mathrm{c}$ & $0,40 \mathrm{~b}$ & $0,47 \mathrm{~b}$ & $0,71 \mathrm{a}$ \\
\hline \multicolumn{5}{|c|}{ Massa seca do sistema radicular (g/planta) } \\
\hline Controle & $3,08 \mathrm{aA}$ & $5,44 \mathrm{aA}$ & $5,05 \mathrm{aA}$ & $7,58 \mathrm{aA}$ \\
\hline Estressado & $3,26 \mathrm{aA}$ & $4,31 \mathrm{aB}$ & $4,87 \mathrm{aA}$ & $6,06 \mathrm{aB}$ \\
\hline Média & $3,17 \mathrm{c}$ & $4,87 \mathrm{~b}$ & $4,96 \mathrm{~b}$ & $6,82 \mathrm{a}$ \\
\hline \multicolumn{5}{|c|}{ Massa seca da parte aérea (g/planta) } \\
\hline Controle & $7,07 \mathrm{cA}$ & $11,27 \mathrm{bA}$ & $12,65 \mathrm{aA}$ & $15,88 \mathrm{aA}$ \\
\hline Estressado & $6,86 \mathrm{cA}$ & $10,09 \mathrm{bA}$ & $10,07 \mathrm{bB}$ & $12,84 \mathrm{aB}$ \\
\hline Média & $6,97 \mathrm{~d}$ & $10,68 \mathrm{c}$ & $11,36 \mathrm{~b}$ & $14,36 \mathrm{a}$ \\
\hline \multicolumn{5}{|c|}{ Massa seca total (g/planta) } \\
\hline Controle & $10,15 \mathrm{cA}$ & $16,71 \mathrm{bA}$ & $17,70 \mathrm{bA}$ & $23,46 \mathrm{aA}$ \\
\hline Estressado & $10,12 \mathrm{cA}$ & $14,40 \mathrm{bB}$ & $14,94 \mathrm{bB}$ & $19,33 \mathrm{aB}$ \\
\hline Média & $10,14 \mathrm{c}$ & $15,56 \mathrm{~b}$ & $16,32 \mathrm{~b}$ & $21,40 \mathrm{a}$ \\
\hline \multicolumn{5}{|c|}{ Área foliar ( $\left.\mathrm{dm}^{2} / \mathrm{planta}\right)$} \\
\hline Controle & $6,34 \mathrm{cA}$ & 9,36 bA & $9,50 \mathrm{bA}$ & $14,53 \mathrm{aA}$ \\
\hline Estressado & $6,22 \mathrm{cA}$ & $7,87 \mathrm{bcA}$ & 8,16 abB & $9,97 \mathrm{aB}$ \\
\hline Média & $6,28 \mathrm{c}$ & $8,62 \mathrm{~b}$ & $8,83 \mathrm{~b}$ & $12,25 \mathrm{a}$ \\
\hline
\end{tabular}

${ }^{1}$ Médias seguidas de mesma letra minúscula na linha e maiúscula na coluna não diferem significativamente entre si, pelo teste de Tukey, ao nível de 0,05 de probabilidade. 
Estas informações corroboram com os dados aqui apresentados, onde a produção de biomassa das diferentes partes da planta foi fortemente influenciada pelo déficit hídrico. A produção de biomassa seca no final do experimento (44 dias) foi comprometida nas plantas sob déficit hídrico, observando-se reduções de 38, 41, 37 e $38 \%$ na biomassa seca de folha, raiz, caule e total, respectivamente, quando comparadas às plantas controle (Figura 1).

Em meio às características morfológicas, a área foliar é a primeira afetada pelo estresse imposto pela deficiência hídrica, devido ao decréscimo na expansão foliar, podendo ser acompanhado de senescência foliar como forma de redução da área transpiratória. Esses ajustes da planta, como diminuição da altura e da área foliar, são estratégias adaptativas de sobrevivência ao ambiente estressante (ZHANG et al., 2004; DÍAZLOPEZ et al., 2012).

No presente experimento nas produções de Af do tratamento estressado, ocorreram reduções aos 25 e 44 dias de imposição dos ciclos de déficits hídricos e foram equivalentes a 14,1 e $31,4 \%$, respectivamente, em relação ao tratamento controle.

Essa redução da área foliar é descrito por diversos autores (DIAS-FILHO; DAWSON, 1995; GUREVITCH, 2009) em resposta à deficiência hídrica no solo.
Isso ocorre em função da diminuição da taxa de transpiração, promovendo a uma economia de água.

Beltrão et al. (2003), verificaram em plantas de Ricinus communis, cultivar BRS 188 Paraguaçu, sob corte de irrigação, em apenas 6 dias, mostraram redução da área foliar em $60 \%$ em relação às plantas controle, indicando alta sensibilidade dessa espécie ao estresse.

Essas reduções de produtividade primária das plantas jovens de açaizeiros sob déficit hídrico, representadas pelas produções de MSF, MSAC, MSR, MSPA, MST e Af são devidas principalmente às reduções da umidade do solo, que neste trabalho atingiram 64,7 e 75,3\%, com 15 e 25 dias de restrição hídrica, durante o primeiro ciclo e 46,4\%, com 14 dias de déficit hídrico, no segundo ciclo de estresse hídrico, em comparação com a umidade do solo das plantas do tratamento controle. Resultados semelhantes têm sido corroborados por vários autores (BRUNINI; CARDOSO, 1998; NOGUEIRA; CONCEIÇÃO, 2000; CONCEIÇÃO et al., 2004; TAIZ; ZEIGER, 2013), onde são relatados que, a baixa disponibilidade hídrica no solo afeta negativamente o crescimento dos cultivos agrícolas e, é a principal causa da redução da produtividade.

De acordo com Achten et al. (2010), a aplicação de um estresse moderado (40\% 
da capacidade de campo), em plantas de $J$. curcas, reduziu a produção de biomassa total, porém não foi $\mathrm{o}$ suficiente para produzir alterações no padrão de alocação da biomassa, sendo este mecanismo notório apenas em plantas mantidas sem irrigação.

\section{CONCLUSÃO}

Em condições de déficit hídrico, as produções das massas secas de raízes, folhas, folíolos, ráquis, pecíolo e bainha, foram afetadas negativamente a área foliar de plantas jovens de açaizeiros submetidas a ciclos de deficiência hídrica foi reduzida.

Estudos em plantas adultas, tornam-se necessários para verificar se a redução de biomassa na fase jovem, nas plantas sob déficit hídrico, representa efeito negativo na eficiência de produção em frutos de Euterpe oleraceae.

\section{REFERÊNCIAS}

ACHTEN, W. M. J. et al. Biomass production and allocation in Jatropha curcas L. seedlings under different levels of drought stress. Biomass and Bioenergy, p. 1-10, 2010.

BELTRÃO, N. E. et al. Estresse hídrico (deficiência e excesso) e seus efeitos no crescimento inicial da mamoneira, cultivar BRS 188 Paraguaçu. Rev. Bras. Ol. Fibros., Campina Grande, v. 7, n. 2/3, p. 735-741, 2003.
BRUNUNI, O.; CARDOSO, M. Efeito do déficit hídrico no solo sobre o comportamento estomático e potencial de água em mudas de seringueira. Pesq. agropec. bras., v. 33, n. 7, p. 1053-1060, 1998.

CALBO, M.E.R., MORAES, J.A.P.V., 1997. Fotossíntese, condutância estomática, transpiração e ajustamento osmótico de plantas de buriti submetidas a estresse hídrico. Rev. Bras. Fisiol. Veg., v. 9, n. 2, p.117-123.

CALBO, M.E.R., MORAES, J.A.P.V., 2000. Efeitos da deficiência hídrica em plantas de Euterpe oleracea (açaí). Rev. Bras. Bot. v. 23, 225-230.

CONCEIÇÃO, H. E. O. da; MONTEIRO, A. L. C.; FRAZÃO, D. A. C.; VIÉGAS, I. de J._M.; RIPARDO, A. K. da S. Efeito de déficit hídrico no desenvolvimento de progênies de açaizeiro. In: CONGRESSO BRASILEIRO DE FRUTICULTURA, 18., 2004, Florianópolis. Anais... Tecnologia, competitividade, sustentabilidade. Florianópolis: SBF, 2004.

DIAS-FILHO, M.B.; DAWSON, T.E. Physiological responses to soil moisture stress in two Amazonian gap-invader species. Functional Ecology, v. 9, p.213221, 1995.

DIAS-FILHO, M.B. Physiological responses of two tropical weeds to shade: 
II. Leaf gas exchange and nitrogen content.

Pesquisa Agropecuária Brasileira, v. 34, p.953-961, 1999.

DÍAZ-LOPEZ et al. Jatropha curcas seedlings show a water conservation strategy under drought conditions based on decreasing leaf growth and stomatal conductance. Agricutural Water

Management, v. 105, p. 48-56, 2012.

ENDRES, L.; SOUZA, J. L. de; TEODORO, I.; MARROQUIM, P. M. G.; SANTOS, C. M. dos; BRITO, J. E. D. de Gas exchange alteration caused by water deficit during the bean reproductive stage. Revista Brasileira de Engenharia Agrícola e Ambiental, v. 14, n. 1, p. 1116, jan., 2010.

FARIAS NETO, J. T.; VASCONCELOS, M. A. M.; SIlvA, F. C. F. Cultivo, processamento, padronização e comercialização do açaí na Amazônia. Fortaleza: Instituto de Desenvolvimento da Fruticultura e Agroindústria- FRUTAL, 2010. 147 p. (Coleção Curso Frutal Amazônia/ X Flor Pará, 1)

FIGUEIRÔA, J. M.; BARBOSA, D. C. A.; SIMABUKURO, E. A. Crescimento de plantas jovens de Miracrodruon urundeuva Allemão (Anacardiaceae) sob diferentes regimes hídricos. Acta Botânica Brasílica, v. 18, n. 3, p. 573-580, 2004.
GOMES, F. P., OLIVA, M. A., MIELKE, M. S., ALMEIDA, A-A. F., LEITE, H. G., AQUINO, L. A. Photosynthetic limitations in leaves of young Brazilian Green Dwarf coconut (Cocos nucifera L.'nana') palm under well-watered conditions or recovering from drought stress. Environmental and Experimental Botany. v. 62, p. 195-204. 2008.

GURECITH J., SCHEINER S.M., FOX, G.A. Ecologia Vegetal. Artmed, 2009. 592p.

INSTITUTO BRASILEIRO DE GEOGRAFIA E ESTATÍSTICA. Sidra. 2012. Disponível em: <www.sidra.ibge.gov.br>. Acesso em: 29 março 2013.

JALEEL, C. A., MANIVANNAN, P., WAHID, A., FAROOQ, M., AL-JUBURI, H. J. SOMASUNDARAM, R., PANNEERSELVAM, R., 2009. Drought Stress in Plants: A Review on Morphological Characteristics and Pigments Composition. International Journal Agricultural Biology, v. 11, p. 100-105.

JARDIM, M. A. G. A cadeia produtiva do açaizeiro para frutos e palmitos: implicações ecológicas e socioeconômicas no Estado do Pará. Bol. Mus. Para. Emílio Goeldi, sér. Antropol. v. 18, n. 2, p. 287-305. 2002. 
LECHINOSKI, A. et al. Influência do estresse hídrico nos teores de proteínas e aminoácidos solúveis totais em folhas de Teca (Tectona grandis L. f.). Revista Brasileira de Biociências, Porto Alegre. v. 5, supl.2, p. 927-929, jul. 2007.

LORENZI, H.; SOUZA, H. M.; COSTA, J. T. M.; CERQUEIRA, L. S. C.; BEHR, N. Palmeiras do Brasil: nativas e exóticas. Editora Plantarum. Nova Odessa, 1996. $156 \mathrm{p}$.

MACHADO, A.V. Efeitos do estresse hídrico em plantas jovens de Hedyosmum brasiliense Mart (Choranthaceae). 2004. 59f. Dissertação (Mestrado em Biologia Vegetal) Universidade Federal de Santa Catarina, Florianópolis, 2004.

NOGUEIRA, O. L.; CONCEIÇÃO, H. E. O. Análise de crescimento de açaizeiros em áreas de várzea do estuário Amazônico. Pesquisa Agropecuária

Brasileira, v. 35, p. 2167-2173, 2000.

OLIVEIRA, M. A. J., BOVI, M. L. A., MACHADO, E. C., GOMES, M. M. A., HABERMANN, G., RODRIGUES, J. D., Fotossíntese, condutância estomática e transpiração em pupunheira sob deficiência hídrica. Sci. Agric. v. 59, p. 59-63. 2002.

REPELLIN, A., DANIEL, C., ZUILYFODIL, Y. Merits of physiological tests for characterizing the performance of different coconut varieties subjected to drought. Oleagineux. v. 49, p. 155-167. 1994.

RIPARDO, A. K. da S. Efeito do déficit hídrico no desenvolvimento de progênies de açaizeiro. In: CONGRESSO BRASILEIRO DE FRUTICULTURA, 18. 2004. Anais... Florianópolis: SBF, 2004. p.1-4.

SILVA, M. A. V.; NOGUEIRA, R. J. M. C.; OLIVEIRA, A. F.; SANTOS, V. F. Resposta estomática e produção de matéria seca em plantas jovens de aroeira submetidas a diferentes regimes hídricos. Revista Árvore, Viçosa-MG, v. 32, n. 2, p. 335-344, 2008.

SMIT, J. Root growth and water use efficiency of douglas - fir (Pseutsuga menziestri Mirb. Franco) and lodgepole pine (Pinus contorta Dougl.) seedlings. Tree Physiology, Victoria, v. 11, p. 401410, 1992.

StatSoft Inc， 2006. STATISTICA (data analysis software system), version 8.0.

SUFRAMA - Superintendência da Zona Franca de Manaus. Potencialidades Regionais, Estudo de Viabilidade Econômica: Palmito de Pupunha. Manaus, v. 7, 2003. 36p.

TAIZ, L.; ZEIGER, E. Fisiologia vegetal. Tradução Eliane Romanato Santarém et al. 3.ed. Porto Alegre: Artmed, 2013. 719p. 
TINOCO, A. C. Açaí amazônico: novas perspectivas de negócio. Belém, PA: Embrapa Amazônia Oriental, 2005. 1 CDROM. In: WORKSHOP REGIONAL DO AÇAIZEIRO: PESQUISA, PRODUÇÃO

E COMERCIALIZAÇÃO. Belém, PA, 2005.

VIÉGAS, I. J. M.; FRAZÃO, D. A. C.; THOMAZ, M. A. A.; CONCEIÇÃO, H. E. O. DA; PINHEIRO, E. Limitações nutricionais para o cultivo do açaizeiro em LATOSSOLO AMARELO textura média, Estado do Pará. Revista Brasileira de Fruticultura, v. 26, n. 2, p. 382-384, 2004. XAVIER, L. N. B.; OLIVEIRA, E. A. D. A. Q.; OLIVEIRA, A. L. Extrativismo e manejo do açaí. In: ENCONTRO LATINO AMERICANO DE INICIAÇÃO CIENTÍFICA, 13., ENCONTRO LATINO AMERICANO DE PÓSGRADUAÇÃO, 9., $\quad 2009 . \quad$ Anais... Universidade do Vale do Paraíba, 2009.

ZHANG, W. L.; LI, L. H.; ZU, Y. G.; PEREZ, S. Effect of priming on the germination of Peltophorum dubium seeds under water stress. Journal of Forestry Research, Japão, v. 15, n. 4, p. 287-290, 2004. 